\title{
Pulmonary MALT lymphoma - when an opacity persists too long: a case report and literature review
}

\author{
R. Martins Natal'1', G. Cintrão Samouco', M. Oliveira', L. Vaz Rodrigues ${ }^{1,2}$ \\ ${ }^{1}$ Hospital Sousa Martins, Unidade Local de Saúde da Guarda, Guarda, Portugal; ' 2 Faculdade de Ciências da Saúde da Universidade da Beira Interior, \\ Covilhã, Portugal
}

\begin{abstract}
Mucosa-associated lymphoid tissue (MALT) lymphoma is an extranodal lymphoma arising in a number of epithelial tissues, including stomach, salivary gland and the lung. Pulmonary MALT lymphoma originates in the parenchyma and/or bronchi and is a rare disease, representing 0.5-1\% of primary pulmonary malignancies. Although the time to diagnosis can be delayed because most patients are asymptomatic and investigations are usually driven by the accidental finding of abnormal lung imaging studies, it has an indolent course and a good prognosis. Here we report the case of an asymptomatic female patient with a nodular lung opacity that was found to persist five months after an episode of community-acquired pneumonia. A final diagnosis of pulmonary MALT lymphoma was obtained after an extensive diagnostic work-up.
\end{abstract}

Keywords: Lymphoma, B-Cell, Marginal Zone, Lung Neoplasms

\section{Introduction}

Pulmonary MALT (pMALT) Iymphoma is a clonal lymphoproliferative disorder that arises from B lymphocytes in the epithelial lung tissue. By definition, it affects one or both lungs, without extrapulmonary involvement at diagnosis or during the subsequent three months. It usually has an indolent course and carries a good prognosis. Since it's a silent disease in over $50 \%$ of patients, the mean time to diagnosis is around 9 months ${ }^{1}$. Tissue biopsy is the gold standard for diagnosis ${ }^{1}$. There is no clear consensus on treatment: localized disease can be treated with radiotherapy, surgery or observation while diffuse lymphomas are almost always managed with chemotherapy, either with a single or multiple agents ${ }^{1,2}$.

\section{Case report}

a 72-year old woman was admitted in another hospital emergency with a clinical presentation of a flu-like symptoms that started in the previous 3 days, accompanied by fever, cough and sputum. No changes on clinical examination were found; pulmonary auscultation revealed a vesicular breath sounds on both lungs. Laboratory tests revealed leukocytosis with neutrophilia, mild normocytic normochromic anemia and elevated reactive $\mathrm{C}$ protein. Chest $\mathrm{x}$-ray showed a consolidation of the middle lobe and she was then hospitalized with the diagnosis of a community-acquired middle lobe pneumonia treated to clinical and laboratory resolution with amoxicillin-clavulanic acid and azithromycin. Her chest $x$-ray of discharge still showed the alveolar opacity in the middle lobe.

During follow-up, she had been always asymptomatic. However, her repeated chest $\mathrm{x}$-rays remained showing the alveolar opacity in the middle lobe (Figure 1). A thorax computed tomography (CT), five months after discharge, confirmed the consolidation of the middle lobe (Figure 2) and also revealed another homolateral nodular lesion with $11,5 \mathrm{~mm}$ and multiple hepatic hypodense nodules. At this point she was referred to our pulmonary outpatient clinic where we started her diagnostic work-up. The patient was non smoker and denied previous history of any respiratory illness. She had a history of hypertension, type 2 diabetes, dyslipidemia and vertigo syndrome. On admission, she was asymptomatic, revealed slightly pale mucous membranes and faint symmetrical lower limb edema; otherwise normal. Laboratory tests showed mild normochromic normocytic anemia and mild leukocytosis. HIV (1 and 2) and hepatitis serologies were negative. Auto-immune serum screening was negative.
Fig. 1. A thorax computed tomography (CT), five months after discharge, confirmed the consolidation of the middle lobe (Figure 2) and also revealed another homolateral nodular lesion with $11,5 \mathrm{~mm}$ and multiple hepatic hypodense nodules. At this point she was referred to our pulmonary outpatient clinic where we started her diagnostic work-up.
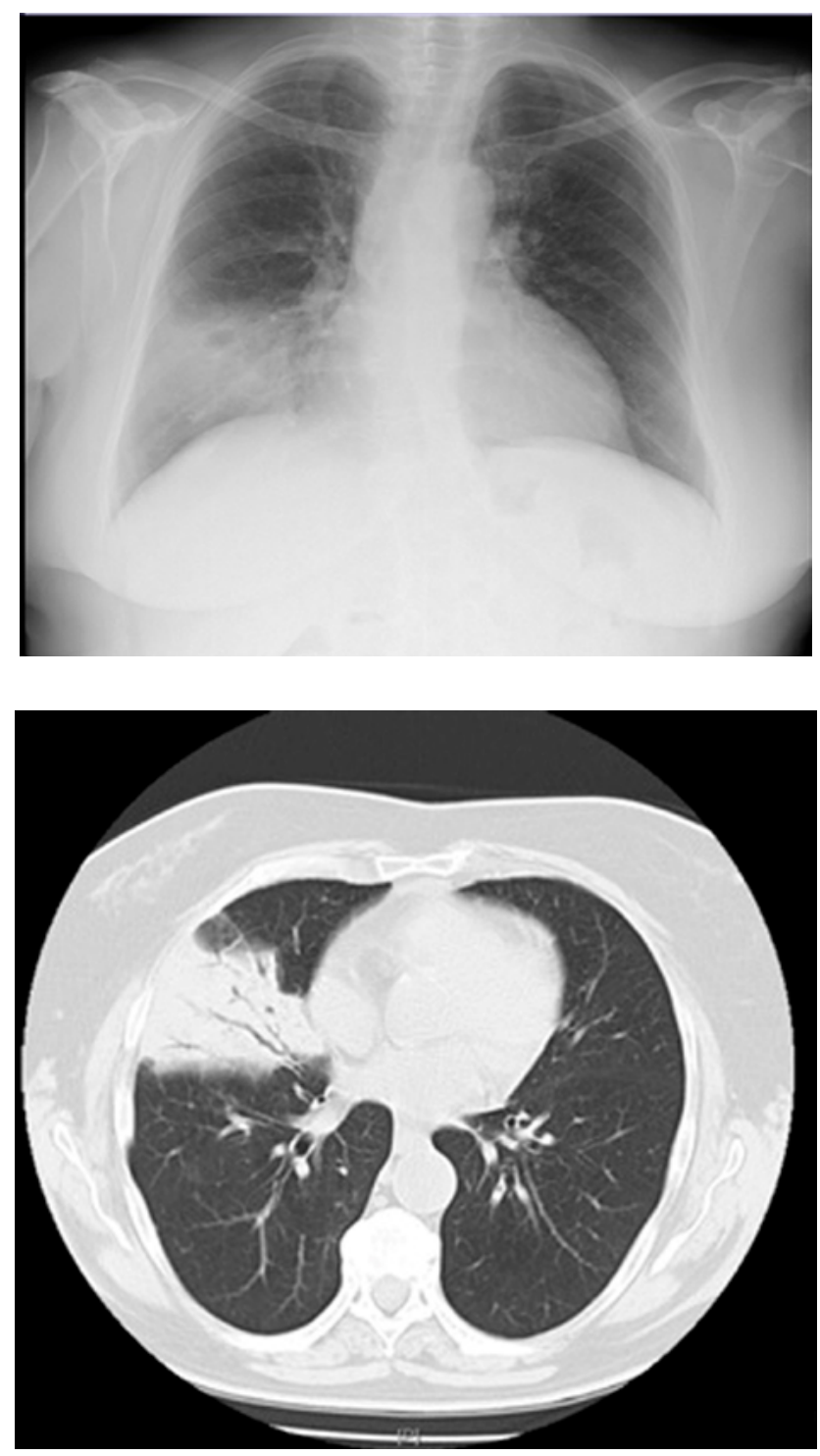
An abdominal ultrasound favored the cystic nature of the hepatic lesions. Fiberoptic bronchoscopy revealed a diffuse mildly friable and inflamed mucosa with a slight enlargement of the right basal pyramid spurs. Bronchoalveolar lavage (BAL) was negative for malignant cells and microbiology analysis but showed marked lymphocytosis (91\%) with a population of monoclonal B lymphocytes. The patient then underwent a CT-guided needle biopsy of the middle lobe lesion that suggested MALT hyperplasia with low proliferative index (Ki67<5\%). A surgical lung biopsy of both the middle lobe mass and right inferior lobe nodule confirmed the diagnosis of PMALT Iymphoma, revealing enlargement of the interalveolar septa due to lymphoid cell proliferation, with positivity for CD20 and Bcl-2 and negativity for CD10, Bcl-6, cyclinD1 and MUM1 at immunohistochemical staining. Bone marrow biopsy presented no evidence of neoplastic involvement.

Subsequently, the patient was transferred to the Hematology Department where she performed a contrast enhanced CT scan of the abdomen and pelvis for staging that failed to reveal further lesions. The diagnosis of pMALT lymphoma (Ann-Arbor satge IE) was established and the patient was started on chemotherapy with R-CVP ( $R$ - rituximab; CVP - cyclophosphamide, vincristine and prednisone).

\section{Discussion}

Pulmonary MALT lymphoma is as a subtype of non-Hodgkin lymphomas that is characterized by a low-grade extranodal marginal zone B-cell proliferation, arising in a number of epithelial tissues, including stomach, salivary gland, lung, small bowel and thyroid. It can also be described as the most common primary pulmonary lymphoma (up to $80 \%$ ), nevertheless a rare disease, representing $0.5-1 \%$ of primary pulmonary malignancies ${ }^{1,3}$.

Increasing evidence suggests that this lymphoma is related to chronic immune stimulation due to bacterial, viral or autoimmune stimuli. The most known studied association is between Helicobacter pylory infection and gastric MALT Iymphoma, not only in the pathogenesis but also in the complete remission when the bacteria are eradicated by antibiotics ${ }^{4}$. However a causative agent or autoimmune process associated to pMALT hasn't been found yet. In our case we can postulate that an infection could have been the trigger since the patient was initially treated to community acquired pneumonia. Unfortunately no microbiological study was performed.

The median age at diagnosis is 50-60 years old and there is no gender predominance ${ }^{3,5}$. Active or former tobacco use is not higher than in general population'. Clinically, half of the cases are asymptomatic and features of autoimmune diseases is present in $16 \%$ of the patients at diagnosis ${ }^{1,5}$.

Patients with PMALT Iymphoma typically present as a chronic alveolar localized opacity less than $5 \mathrm{~cm}$ in diameter and associated with air bronchograms in nearly $50 \%$ of the cases, such as the case we present (Figure 1) $)^{1,3,6}$. The commonest CT findings are single or multiple nodules or areas of consolidation (>70\%), multiple bilateral lesions (>70\%) that tend to be peribronchovascular with intact bronchial lumen (Figure 4) $)^{6}$. Less commonly, hilar and/or mediastinal lymphadenopathy (30\%), bronchiectasis and bronchiolitis, diffuse interstitial lung disease and pleural effusion are found ${ }^{1,6}$. The time between clinical or radiological findings and diagnosis varies from 15 days to 8 years, mean 9 months, which was the exact time from the pneumonia episode to the surgical biopsy result't .
Tissue biopsy is the gold standard for diagnosis'. It may be obtained via minimally invasive procedures, including bronchoscopy or CT-guided needle biopsy, but surgical lung biopsy is sometimes necessary to clarify the diagnosis. In our patient surgical lung biopsy due to its wider representation was fundamental to clarify the diagnosis. Bronchoalveolar lavage (BAL) may aid in the differential diagnosis of chronic alveolar opacitites by revealing the absence of tumor epithelial cells and usually the presence of lymphocytic alveolitis preferably with monoclonal populations (lymphocytes $>20 \%$ of total cells) $)^{3,7}$. In our case we highlight the value of this procedure that was actually the first exam leading to the suspicion of this type of malignancy.

Initial staging includes CT scanning of the chest, abdomen, and pelvis, with contrast injection, for the Ann-Arbor staging system, as was done in our patient. Bone marrow biopsy may show involvement in $13-30 \%$ of cases ${ }^{1,5}$.

There are many therapeutic options but no standard guidelines, owing to the rarity of this lymphoma. For localized disease, most frequently, radiotherapy is the first-line treatment ${ }^{2,9}$. Other options include surgical resection or "watchfull waiting" strategies for asymptomatic patients without cytopenias. In the specific case of localized pulmonary lymphoma, chemotherapy can also be considered, as happened to be the case of our patient, due to concurrent anemia ${ }^{1,2,9}$. In diffuse disease, chemotherapy is preferred, typically with oral alkylating agents (cyclophosphamide or chlorambucil) or purine analogues (fludarabine, 2CdA), or combination chemoterapy (CHOP - cyclophosphamide, adriamycin, vincristine and prednisone; CVP). Rituximab, anti-CD20 monoclonal antibody, has shown efficacy as an additional option to chemotherapy schemes².

Prognosis is good, with 5-year overall survival of over $80 \%$ and a median survival of over 10 years ${ }^{1,9}$. Follow-up is required for a long time as almost $50 \%$ show recurrence of the disease and histological transformation to a more aggressive lymphoma can occur in about $10 \%$ of cases $^{2,9}$.

\section{References}

1. Borie R, Wislez M, Antoine M, Cadranel J. Lymphoproliferative Disorders of the Lung. Respiration. 2017; 94(2): 157-175

2. M Joshi, H Sheikh et al.: Marginal zone lymphoma: old, new, targeted, and epigenetic therapies. Ther Adv Hematol 2012; 3(5): 275 -290

3. Cadranel J, Wislez M, Antoine M: Primary pulmonary lymphoma. Eur Respir J 2002; 20: 750-762.

4. Suarez F, Lortholary 0 , Hermine 0 , Lecuit M. Infection-associated lymphomas derived from marginal zone $B$ cells: a model of antigen-driven lymphoproliferation. Blood. 2006;107(8):3034-3044.

5. Borie R, Wislez M, Thabut G, Antoine M, Rabbat A, Couderc LJ, Monnet I, Nunes H, Blanc FX, Mal H, Bergeron A, Dusser D, Israël-Biet D, Crestani B, Cadranel J: Clinical characteristics and prognostic factors of pulmonary MALT Iymphoma. Eur Respir J. 2009; 34: 1408-1416.

6. Hare S, Souza C, Bain G, Seely J, Frcpc, Gomes M et al. The radiological spectrum of pulmonary lymphoproliferative disease. The British Journal of Radiology. 2012;85(1015):848-864.

7. Drent M, Wagenaar SS, Mulder PH, van Velzen-Blad H, Diamant M, van den Bosch JM. Bronchoalveolar lavage fluid profiles in sarcoidosis, tuberculosis, and non-Hodgkin's and Hodgkin's disease. An evaluation of differences. Chest 1994; 105: 514-519

8. Agarwal KK, Dhanapathi H, Nazar AH, Kumar R. Primary pulmonary lymphoma-role of fluoro-deoxy-glucose positron emission tomography-computed tomography in the initial staging and evaluating response to treatment - case reports and review of literature. Indian Journal of Nuclear Medicine. 2016;31(3):194.

9. de Boer J, Hiddink R, Raderer M, Antonini N, Aleman B, Boot $\mathrm{H}$ et al. Dissemination patterns in non-gastric MALT lymphoma. Haematologica. 2008;93(2):201-206.

10. Parissis H. Forty years literature review of primary lung lymphoma. Journal of Cardiothoracic Surgery. 2011;6(1). 